\title{
Analysis and Design of EMI Filter for Multi-Output Switching Mode Power Supply
}

\author{
Zong-Yu He*, Fu-Yuan Shih*, Yie-Tone Chen** and Yan-Pei Wu* \\ * Department of Electrical Engineering \\ National Taiwan University \\ Taipei, Taiwan, R.O.C. \\ ** Department of Electrical Engineering \\ National Yun-Lin Institute of Technology \\ Tou-Liu City, Yun-Lin County, Taiwan, R.O.C.
}

\begin{abstract}
In this paper, a generalized method has been used to discuss the influence of the EMI filter on the stability and the performance of multi-output switching mode power supplies. A criteria for the design of single stage EMI filter has been proposed. This criteria will satisfy the requirement of specification in EMI and will guarantee the system to be stable at the same time. Furthermore, whether there exists the post regulator or preregulator in the system or a single output system, the generalized formula of performance derived in this paper will work as well.
\end{abstract}

\section{Introduction}

Electromagnetic Interference(EMI) has become the major problem for the designers and manufactures of switching mode power supply in recent years. Because the EMI noise caused by the discontinuous switching current ripple of a switching power supply is always over the noise immunity of the other equipments, it has become the most important part in the design of switching power supply to reduce such kind of high frequency noise and make the product to meet the requirement of EMI specification. To overcome this problem, a lowpass filter, so-called EMI filter or input filter, is usually added in front of the switching power stage. However, when the EMI filter was utilized, it lead to the degradation effect of the characteristics of switching power supply, even cause the instability of the overall system. The situation will become more serious when the designers apply the topology of multi-output power supplies. The reason is that the multi-output scheme is often used to design higher power and meets more other requirements. If the multi-output power supplies consist of postregulators, the problem will become more complicated.

In this paper, the affection of EMI filter to the stability and performance of the multi-output forward power converters will be discussed. The reason of instability will be presented and the design criteria of EMI filter will be also discussed. After the criteria have been met, the designers can make their products meet the requirement of safety and guarantee the stable operation. Furthermore, the generalized formulas of performance will be derived, so whether there exist the post regulators in the system or for a single-output system, the formulas will work as well. Then, the results of simulation and experiment will be presented. Section V concludes the paper.

\section{Small-Signal Model of Two-Output Forward Converters}

Figure 1 shows the circuit diagram of a two-output forward converter. The main output is regulated through the duty ratio control of the switch S1 and the auxiliary output is regulated by the post regulator. The detail of the control transfer function of Fig. 1 will be derived below.

According to the different conduction state of main switch S1 and the switch S2 of post regulator(Fig.2), the two-output converter will have the following three different conduction periods: (1) Switch 1 is in conduction and switch 2 is opened (2) Switch 1 and switch 2 are both in conduction (3) Switch 1 and switch 2 are both opened. Fig. 3 shows the equivalent circuit diagram of the above three different conduction periods. By the principle of state-space avering[1], the control transfer function of Fig. 1 can be derived and is summarized in Table I.

From the results of Table I, if one makes $D 2=0$ and $\mathrm{T} 2=0$, the situation will like that without a post regulator. On the contrary, if one makes $D 2=D 1$, then the situation will like that of single output. Thus Table I is also applied to two-output forward converters without post regulator and the single-output forward 


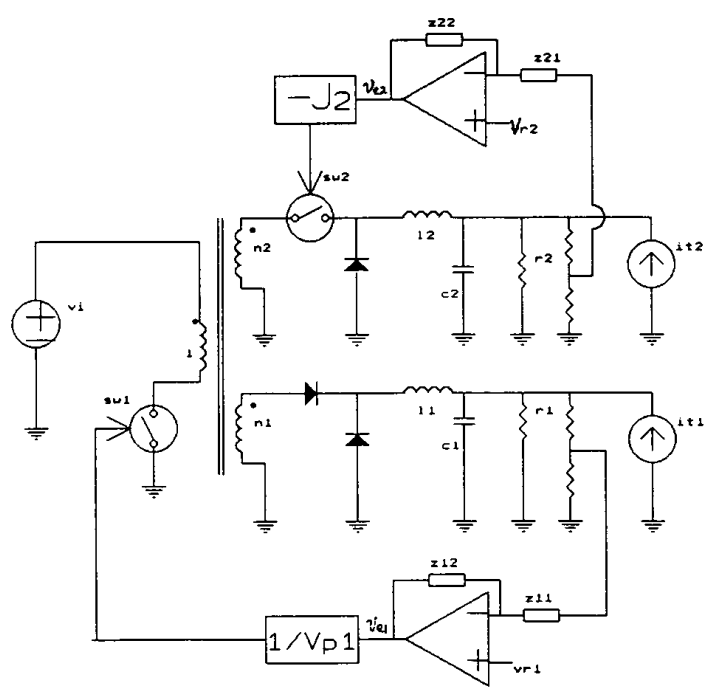

Figure 1 The circuit diagram of a two-output forward converter with post-regulator

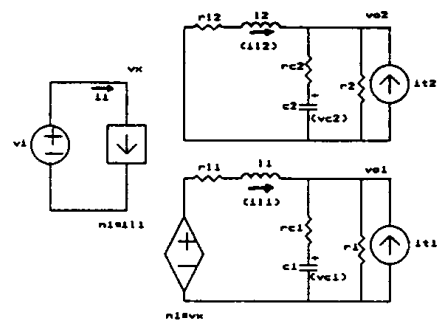

(a)

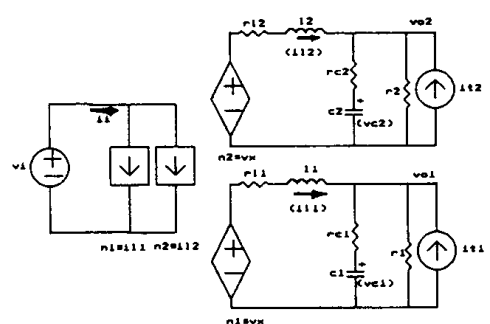

(b)

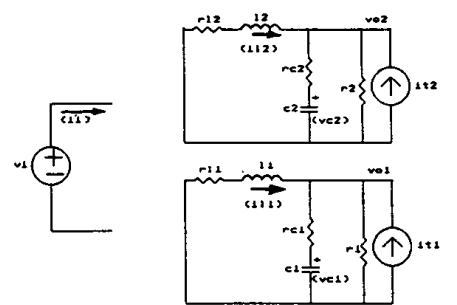

(c)

Figure 2 The three different states of a period (a)sw1-on, sw2-off (b)sw1-on, sw2-on (c)sw1-off, sw2-off
Table I Transfer Functions of Two-Output Forward Converters with Post Regulator

$$
\begin{aligned}
& \frac{\hat{i}_{i}}{\hat{v}_{i}}=t_{11}=-\left(\frac{D_{1}^{2} n_{1}^{2}}{r 1}+\frac{D_{1}\left(D_{1}-D_{2}\right) n_{2}^{2}}{r 2}\right)\left(\frac{T_{1}}{1+T_{1}}\right) \\
& +\left(\frac{D_{1}^{2} n_{1}^{2}}{r 1}\right)\left(\frac{\frac{r l}{L 1} S+\frac{1}{L i C 1}}{\Delta_{1}}\right)\left(\frac{1}{1+T_{1}}\right)-\frac{\left(D_{1}-D_{2}\right)^{2} n_{2}^{2}}{r 2}\left(\frac{T_{2}}{1+T_{2}}\right) \\
& +\frac{D_{1}\left(D_{1}-D_{2}\right) n_{2}^{2}}{r_{2}}\left(\frac{T_{1}}{1+T_{1}}\right)\left(\frac{T_{2}}{1+T_{2}}\right) \\
& +\frac{\left(D_{1}+D_{2}\right)^{2} n_{2}^{2}}{r_{2}}\left(\frac{\frac{n}{L^{2}} S+\frac{1}{L 2 C 2}}{\Delta_{2}}\right)\left(\frac{1}{1+T_{2}}\right) \\
& -\frac{D_{1}\left(D_{1}-D_{2}\right) n_{2}^{2}}{r^{2}}\left(\frac{\frac{2}{L} S+\frac{1}{L 2 C}}{\Delta_{2}}\right)\left(\frac{T_{1}}{1+T_{1}}\right)\left(\frac{1}{1+T_{2}}\right) \\
& \frac{\hat{v_{01}}}{\hat{v_{i}}} \triangleq t_{21}=n_{1} D_{1}\left(\frac{\frac{\mathrm{rel}}{L 1} S+\frac{1}{\mathrm{LIC1}}}{\Delta_{1}}\right)\left(\frac{1}{1+T_{1}}\right) \\
& \frac{\hat{v_{o 2}}}{\hat{v_{i}}} \stackrel{\Delta}{=} t_{31}=n_{2}\left(D_{1}-D_{2}\right)\left(\frac{\frac{c 2}{L L} S+\frac{1}{L 2 C 2}}{\Delta_{2}}\right)\left(\frac{1-\frac{D_{2}}{D_{1}-D_{2}} T_{1}}{1+T_{1}}\right)\left(\frac{1}{1+T_{2}}\right) \\
& \frac{\hat{i}_{1}}{\hat{i_{1}}} \triangleq t_{12}=-\frac{D_{1} n_{1} r c 1}{L \mathrm{l}}\left(\frac{S+\frac{1}{\text { ricl }}}{\Delta_{1}}\right)\left(\frac{1}{1+T_{1}}\right)-D_{1} n_{1}\left(\frac{T_{1}}{1+T_{1}}\right) \\
& -\left(\frac{D_{1} L_{1}}{r 1}+\frac{\left(D_{1}-D_{2}\right) n_{2} L_{2}}{n_{1} r_{2}}\right) S \frac{T_{1}}{1+T_{1}} \\
& -\frac{\left(D_{1}-D_{2}\right) n_{2}^{2} L_{1}}{n_{1} r^{2}} S\left(\frac{T_{1}}{1+T_{1}}\right)\left(\frac{T_{2}}{1+T_{2}}\right) \\
& -\frac{\left(D_{1}-D_{2}\right) n_{2}^{2} L_{1}}{n_{1} L_{2}}\left(\frac{S+\frac{1}{r 2 C_{2}}}{\Delta_{2}}\right) S\left(\frac{T_{1}}{1+T_{1}}\right)\left(\frac{1}{1+T_{2}}\right) \\
& \frac{\hat{i_{i}}}{\hat{n}} \stackrel{\Delta}{=} t_{13}=-\frac{\left(D_{1}-D_{2}\right) n_{2} r_{c 2}}{L_{2}}\left(\frac{S+\frac{1}{r_{c 2} C_{2}}}{\Delta_{2}}\right)\left(\frac{1}{1+T_{2}}\right) \\
& \frac{\left(D_{1}-D_{2}\right) n_{2} L_{2}}{r_{2}}\left(S+\frac{r_{2}}{L_{2}}\right)\left(\frac{T_{2}}{1+T_{2}}\right) \\
& \frac{\hat{v_{02}}}{\hat{i_{11}}} \stackrel{\Delta}{=} t_{32}=-\frac{n_{2} L_{1} r_{c 2}}{n_{1} L_{2}}\left(\frac{S+\frac{1}{r_{c 2} c_{2}}}{\Delta \Delta_{2}}\right) S\left(\frac{T_{1}}{1+T_{1}}\right)\left(\frac{1}{1+T_{2}}\right) \\
& \frac{\hat{\nu_{0}}}{\hat{i}} \stackrel{\Delta}{=} t_{22}=\frac{1}{C_{1}}\left(\frac{S}{\Delta \Delta_{1}}\right)\left(\frac{1}{1+T_{1}}\right) \\
& \frac{\hat{v}_{02}}{\hat{i}} \stackrel{\Delta}{=} t_{33}=\frac{1}{C_{2}}\left(\frac{S}{\Delta}\right)\left(\frac{1}{1+T_{2}}\right) \\
& \frac{\hat{v_{11}}}{\hat{i_{2}}}=t_{23}=0 \\
& G_{d 1} \triangleq \frac{\hat{v_{01}}}{\hat{d}}=\frac{n_{1} V_{l}\left(r_{c 1} C_{1} S+1\right)}{L_{1} C_{1} \Delta_{1}} \\
& G_{d 2} \triangleq \frac{\hat{v_{02}}}{\hat{d}}=\frac{n_{2} V_{r}\left(r_{2} C_{2} S+1\right)}{L_{2} C_{2} \Delta 2}
\end{aligned}
$$

Where $\quad \Delta_{1}=S^{2}+\left(\frac{r_{c l}}{L_{1}}+\frac{1}{r_{1} c_{1}}\right) S+\frac{1}{L_{1} C_{1}}$

$$
\Delta_{2}=S^{2}+\left(\frac{r_{c_{2}}}{L_{2}}+\frac{1}{r_{2} C_{2}}\right) S+\frac{1}{L_{2} C_{2}}
$$

$$
\begin{aligned}
& T_{1}=K_{1}(S) F_{m 1} G_{d 1} ; K_{1}(S)=\frac{Z_{12}(S)}{Z_{11}(S)}, F_{m 1} \triangleq \frac{\hat{d}_{1}}{v_{e_{1}}}=\frac{1}{V_{p}} \\
& T_{2}=K_{2}(S) F_{m 2} G_{d 2} ; K_{2}(S)=\frac{Z_{22}(S)}{Z_{21}(S)}, F_{m 2} \triangleq \frac{\hat{d_{2}}}{v_{v_{2}}}=J_{2}
\end{aligned}
$$


converters. The input admittance $\frac{\hat{i}_{i}}{\hat{v}_{i}}$ in Table I will be negative when $\mathrm{T} 1$ and $\mathrm{T} 2$ are much larger than one. Because of the characteristic of the negative impedance, when the input filter is added to the system additionally, the system will have the potential of unstable. About the problem of stability, section III will discuss it briefly.

In ideal case, the load variation of output \#2 will have no affection on the output voltage of output \#1, but the load variation of output $\# 1$ does have the affection for the output voltage of output \#2. The results can be obtained from $\frac{\hat{v_{o 1}}}{\hat{i_{t 2}}}$ and $\frac{\hat{v_{o 2}}}{\hat{i_{t 1}}}$ in Table I. From the expression of $\frac{\hat{v_{o 2}}}{\hat{v_{i}}}$, if $\mathrm{T} 1$ can be made to equal to $\frac{D_{2}}{D_{1}-D_{2}}$, then the output \#2 will not be influenced by the input interference in theory, but in practice, $\mathrm{T} 1$ cannot be kept to be constant.

\section{The Switching Power Supply with EMI Filter}

(1)Audiosusceptibility Consideration

EMI filter used in switching power supply(SPS) is shown in Figure 3. From the theorem of reciprocity, one can obtain

$$
\frac{\hat{v_{n}}}{\hat{v_{n}}}=\frac{\hat{i_{n}}}{\hat{i_{n}}}=H_{s}(s)
$$

Because the requirement of EMI filter is often a low-pass filter, so $\mathrm{Z} 2(\mathrm{~s})$ is mainly composed of resistive and capacitive elements, and $Z 1(\mathrm{~s})$ is composed of resistive and inductive elements in practice. Figure 3 can then be equivalent to Figure 4 by the Thevenin theorem. From Figure 4 and Table I, the variation of input voltage-to-output voltage for the two-output forward converters with EMI filter can be derived and is summarized in the following.

$$
\begin{aligned}
\frac{\hat{v_{o 1}}}{\hat{\hat{v}}}= & D_{1} n_{1}\left(\frac{\frac{r_{c 1}}{L_{1}} s+\frac{1}{L_{1} C_{1}}}{\Delta_{1}}\right)\left(\frac{1}{1+T_{1}}\right) H_{s}(s) \bullet \\
& \left(\frac{1}{1+Z_{s}(s) / Z_{i}(s)}\right) \\
\frac{\hat{v_{o 2}}}{\hat{\hat{v}}}= & \left(D_{1}-D_{2}\right) n_{2}\left(\frac{\frac{r_{c 2}}{L_{2}} s+\frac{1}{L_{2} C_{2}}}{\Delta_{2}}\right)\left(\frac{1}{1+T_{2}}\right) \bullet
\end{aligned}
$$

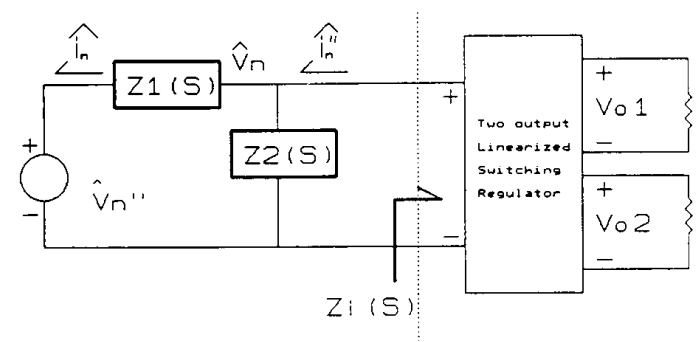

Figure 3 EMI filter and two-output linearized switching regulator

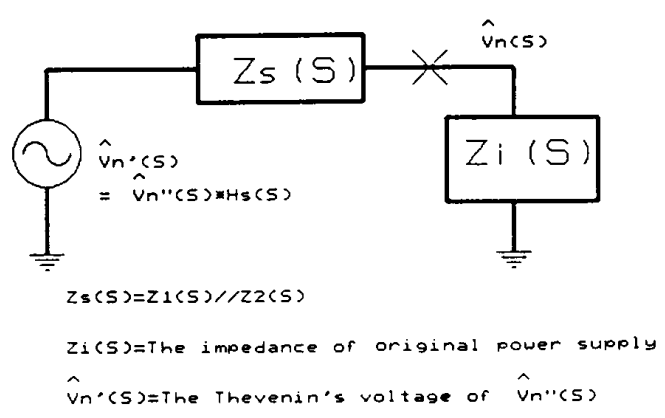

Figure 4 EMI filter and equivalent input impedance of SPS

$$
H_{s}(s)\left(\frac{1}{1+Z_{s}(s) / Z_{i}(s)}\right)\left(\frac{1-\frac{D_{2}}{D_{1}-D_{2}} T_{1}}{1+T_{1}}\right)
$$

Let

$$
\begin{aligned}
& G_{v 1}(s)= D_{1} n_{1}\left(\frac{\frac{r_{c 1}}{L_{1}} s+\frac{1}{L_{1} C_{1}}}{\Delta_{1}}\right) \\
& G_{v 2}(s)=\left(D_{1}-D_{2}\right) n_{2}\left(\frac{\frac{r_{c 2}}{L_{2}} s+\frac{1}{L_{2} C_{2}}}{\Delta_{2}}\right) \bullet \\
&\left(\frac{1-\frac{D_{2}}{D_{1}-D_{2}} T_{1}}{1+T_{1}}\right)
\end{aligned}
$$

Assume that $\mathrm{Zs}(\mathrm{s})<<\mathrm{Zi}(\mathrm{s})$, then (2) and (3) can be approximated to be

$$
\begin{aligned}
& \hat{\frac{v_{o 1}}{\hat{v}}}=G_{v 1}(s) H_{s}(s) /\left(1+T_{1}\right) \\
& \hat{v_{n}} \\
& \frac{\hat{v_{o 2}}}{\hat{v_{n}^{\prime \prime}}}=G_{v 2}(s) H_{s}(s) /\left(1+T_{2}\right)
\end{aligned}
$$


Where Gv1(s) and Gv2(s) are the uncompensated audiosusceptibility of output \#1 and output $\# 2$.

\section{(2) Stability Consideration}

When a EMI filter is added to a SPS, the performance of the original system may be changed. The characteristic equation of the new system can be proved to be

$$
(1+\mathrm{Zs}(\mathrm{s}) / \mathrm{Zi}(\mathrm{s}))(1+\mathrm{T} 1)(1+\mathrm{T} 2)=0
$$

One can conclude from the above equation that the adding of EMI filter is like to increase a new feedback loop, and the open loop gain is $\mathrm{Zs}(\mathrm{s}) / \mathrm{Zi}(\mathrm{s})$. Assume that the original system is stable, then $(1+\mathrm{T} 1)(1+\mathrm{T} 2)$ will have no right half plane(RHP) roots. Because of the reason, if the following equation has no RHP roots, then the new system with EMI filter can be stable.

$$
1+\mathrm{Zs}(\mathrm{s}) / \mathrm{Zi}(\mathrm{s})=0
$$

Apparently, as long as $\left|Z_{s}(s)\right| \ll\left|Z_{i}(s)\right|$, the new system will have not the problem of stability. The conclusion is like that of single output[2]. Before continuing to consider the stability problem, what kind of the input filter belongs to must be determined first. In this paper, a single-stage LC filter(shown in Figure 5 ) is applied. The attenuation function $\mathrm{Hs}(\mathrm{s})$ of this single-stage filter is

$$
H_{s}(s)=\frac{S C_{f} r_{c f}+1}{S^{2} L_{f} C_{f}+S C_{f}\left(r_{d}+r_{c f}\right)+1}
$$

and the equivalent impedance $\mathrm{Zs}(\mathrm{s})$ of this single-stage filter is

$$
Z_{s}(s)=\frac{S L_{f}+r_{d}}{s^{2} L_{f} C_{f}+S C_{f} r_{d}+1}
$$

The resonant frequency of this filter is $w_{p}=\frac{1}{\sqrt{L_{f} C_{f}}}$, and its zero is $w_{z}=\frac{1}{r_{c f} C_{f}}$.

If the attenuation of a system is required to be $\mathrm{A}(-20 \log \mathrm{AdB})$, the restriction of the elements of the

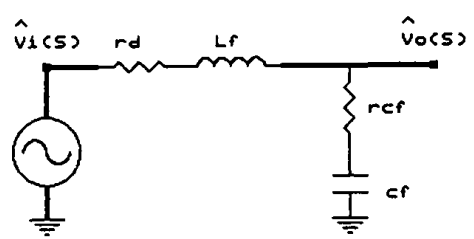

Figure 5 Single-stage lowpass LC filter filter can be achieved according to the relative location of $\mathrm{Wn}, \mathrm{Wp}$, and $\mathrm{Wz}$.

(a) $\mathrm{Wn} \gg \mathrm{Wz}$

$$
\begin{aligned}
& \text { If } C_{f}>\frac{1}{r_{c f} w_{n}} \\
& \text { then } L_{f}>\frac{r_{c f}}{w_{n}} A
\end{aligned}
$$

(b) $\mathrm{Wz} \gg>\mathrm{Wn} \gg>\mathrm{Wp}$

$$
\begin{aligned}
& \text { If } C_{f} \ll<\frac{1}{r_{c f} w_{n}} \\
& \text { then } L_{f}>\frac{A}{w_{n}^{2} C_{f}}
\end{aligned}
$$

The above restriction can be used to determine the value of component at the begining of the filter design.

After the decision of the kind of the filter has been made, the stability will then be discussed according to the different magnitude of loop gains T1 and T2. The discussion can be classified into the following three cases :

(a)T1, T2 $>>1$ : That is, in the region of $\mathrm{W}<<\mathrm{Wb}$ of Figure $6(a)$ or in the region of $W<<W a$ of Figure $6(b)$

$$
\begin{aligned}
\frac{1}{Z_{i}(s)} & =\frac{\hat{i_{i}}}{\hat{v_{i}} \cong}-\left(\frac{n_{1}^{2} D_{1}^{2}}{r_{1}}+\frac{\left(D_{1}-D_{2}\right)^{2} n_{2}^{2}}{r_{2}}\right) \\
& =-\frac{P}{V_{n}^{2}} \\
V_{n} & =\frac{V_{i}+\sqrt{V_{i}^{2}-4 r_{d} P}}{2} \\
r_{d} & \leq \frac{V_{I}^{2}}{4 P}
\end{aligned}
$$

Where $\mathrm{P}$ is the output power of the original power supply, $\mathrm{Vn}$ is the steady-state input voltage, and $\mathrm{Vi}$ is the external input DC source.

Substitution of equations (17), (16), and (11) into equation (9), and according to the rule of Routh-Hurwitz, the another constraints can be obtained to be

$$
\begin{aligned}
f_{1}\left(r_{d}\right)= & r_{d}^{2}-\frac{V_{I}^{2}}{2 P} r_{d}+\frac{L_{f}}{C_{f}}<0 \\
f_{2}\left(r_{d}\right)= & r_{d}^{4}+2\left(\frac{L_{f}}{C_{f}}\right) r_{d}^{2}-\frac{V_{I}^{2}}{P}\left(\frac{L_{f}}{C_{f}}\right) r_{d} \\
& +\left(\frac{L_{f}}{C_{f}}\right)^{2}<0
\end{aligned}
$$



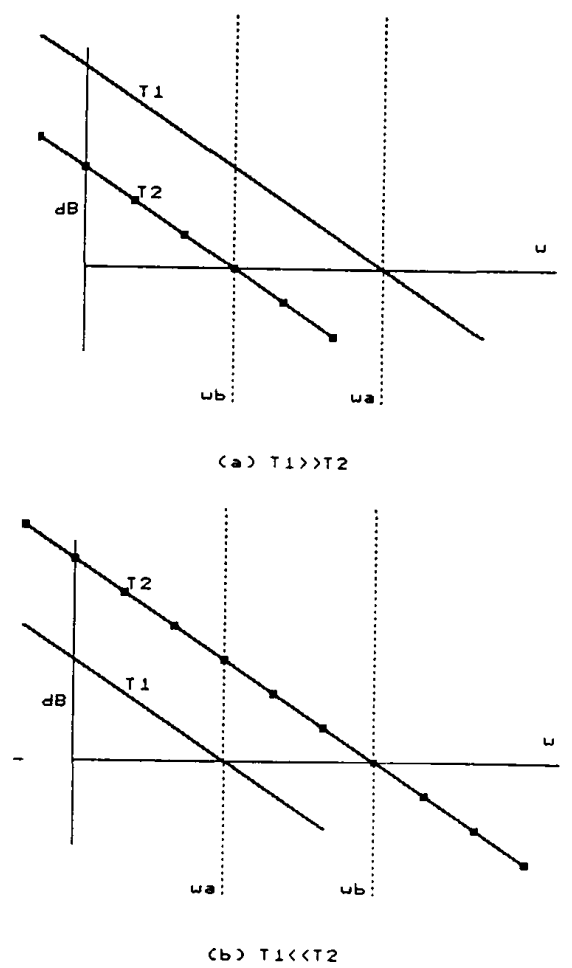

Figure 6 Relations between loop gains $\mathrm{T} 1$ and $\mathrm{T} 2$

(b) T1 $>>1>>$ T2: That is, in the region of $\mathrm{Wb}<<\mathrm{W}<<\mathrm{Wa}$ of Figure $6(\mathrm{a})$

$$
\begin{aligned}
\frac{1}{Z_{i}(s)}= & -\left(\frac{n_{1}^{2} D_{1}^{2}}{r_{1}}+\frac{D_{1}\left(D_{1}-D_{2}\right) n_{2}^{2}}{r_{2}}\right) \\
& -\frac{D_{2}\left(D_{1}-D_{2}\right) n_{2}^{2}}{r_{2}}\left(\frac{\frac{r_{2}}{L_{2}} s+\frac{1}{L_{2} C_{2}}}{\Delta_{2}}\right)
\end{aligned}
$$

If D2 is set to zero, the input admittance of a two-output forward converter without the post regulator can be achieved.

(c) T2 $\gg$ 1 $\gg$ T1: That is, in the region of $\mathrm{Wb}>>\mathrm{W}>>\mathrm{Wa}$ of Figure 6(b)

$$
\frac{1}{Z_{i}(s)} \cong-\left(\frac{\left(D_{1}-D_{2}\right)^{2} n_{2}^{2}}{r_{2}}\right)+\frac{D_{1}^{2} n_{1}^{2}}{r_{1}}\left(\frac{\frac{r_{1}}{L_{1}} s+\frac{1}{L_{1} C_{1}}}{\Delta_{1}}\right)
$$

For cases (b) and (c), the accurate prediction of the constrained value of $\mathrm{rd}$ is difficult. The practical design rule is: (1) avoid the resonant frequency of EMI filter to approach the resonant frequencies of output \#1 and output \#2 (2) check if $\left|\frac{Z_{s}(s)}{Z_{i}(s)}\right|<1$.
(3) Performance Degradation Consideration

After the adding of input EMI filter, the performance of the overall system will be degraded. What affection of performance degradation by the EMI filter can be estimated from Table II. In section IV, the practical simulation and experimental examples can be demonstrated.

Table II Performances of Two-Output Forward Converters with EMI Filter

$t_{11}^{\prime}=H s\left(\frac{t_{11}}{1+t_{11} Z_{s}}\right) ; t_{12}^{\prime}=\frac{t_{12}}{1+t_{11} Z_{s}} ; t_{13}^{\prime}=\frac{t_{13}}{1+t_{11} Z_{s}}$

$t_{21}^{\prime}=H_{S}\left(t_{21}-\frac{t_{21} t_{11} z_{s}}{1+t_{11} z_{s}}\right) ; t_{22}^{\prime}=t_{22}-\frac{t_{12} t_{21} z_{s}}{1+t_{11} Z_{s}} ; t_{23}^{\prime}=-\frac{t_{13} t_{21} z_{s}}{1+t_{11} Z_{s}}$

$t_{31}^{\prime}=H_{S}\left(t_{31}-\frac{t_{31} t_{11} Z_{s}}{1+t_{11} Z_{s}}\right) ; t_{32}^{\prime}=t_{32}-\frac{t_{12} t_{31} Z_{s}}{1+t_{11} Z_{s}} ; t_{33}^{\prime}=t_{33}-\frac{t_{13} t_{31} Z_{s}}{1+t_{11} Z_{s}}$

\section{Simulation and Experimental Results}

The following specification of the two-ouput forward converter with the post regulator is used to simulate various performance of the converter by the Pspice[3,4].

$\mathrm{L} 1=45 \mathrm{uH}, \mathrm{Cl}=1000 \mathrm{uF}, \mathrm{L} 2=90 \mathrm{uH}, \mathrm{C} 2=1000 \mathrm{uF}$, $\mathrm{Fs}=80 \mathrm{kHz}, \mathrm{Vi}=48 \mathrm{~V}, \mathrm{P}=96 \mathrm{~W}$.

To meet the requirement of VDE-0871/B, the attenuation will be $63 \mathrm{~dB}$ at least at the frequency of 80 $\mathrm{kHz}$. According to the criteria of Equations (12), (13), (14), (15), (18), (19), and (20), the single stage EMI filter can be chosen, they are

\section{$\mathrm{Lf}=420 \mathrm{uH}, \mathrm{Cf}=400 \mathrm{uF}$}

The value of $\mathrm{rd}$ can be chosen between $0.05 \Omega$ and $5.7 \Omega$. For the reason that the effect of adding a EMI filter can be easily seen from the simulation, the value of $\mathrm{rd}=0.1 \Omega$ is chosen. After these values have been chosen, the single-stage filter is completely designed. To satisfy the requirement of stability, the conventional compensation method is applied to design a stable output \#1 and output \#2. After the EMI filter has been chosen and the compensation has been designed, whether the overall system is stable or not must be also checked. From Figure 7, the magnitude of $\mathrm{Zs}(\mathrm{s})$ is smaller than $\mathrm{Zi}(\mathrm{s})$, so the overall system can be said to be stable.

Figures 8 to 16 show the simulation results for the various kind of performance of the two-output 
forward converter with EMI filter. From the simulation results, the effect of adding a EMI filter will degrade the performance of SPS can be seen, and the simulation results agree with the calculations from Table II. Figures 8 to 16 also show the amount of degradation is greatly affected by the $Q$ factor of the EMI filter. Comparison of Figures 17, 18, and 14 shows the results of experiment and simulation are in agreement.

\section{Conclusions}

Generalized formula of Two-output forward converters with post regulator have been derived, and the performance degradation of adding a EMI filter to the two-output converters has also been analyzed and discussed. The stability criteria of the converters with EMI filter has been proposed, too. With these guidelines, the stable and high-performance multioutput converters can be achieved.

\section{References}

[1] R. D. Middlebrook and S. Cuk', "A general unified approach to modeling switching converter power stages," in Proc. IEEE Power Electronics Specialists Conf., 1976.

[2] R. D. Middlebrook, " Input filter considerations in design and application of switching regulators," IEEE Industry Applications Society Annual Meeting, 1976 Record.

[3] V. Bello, "Computer aided analysis of switching regulators using SPICE," in IEEE PESC Rec., 1980.

[4] V. Vorperian, "Simplified analysis of PWM converters using the model of the PWM switch:Parts I and II," IEEE Trans. Aerosp. Electron. Syst., Mar. 1990.

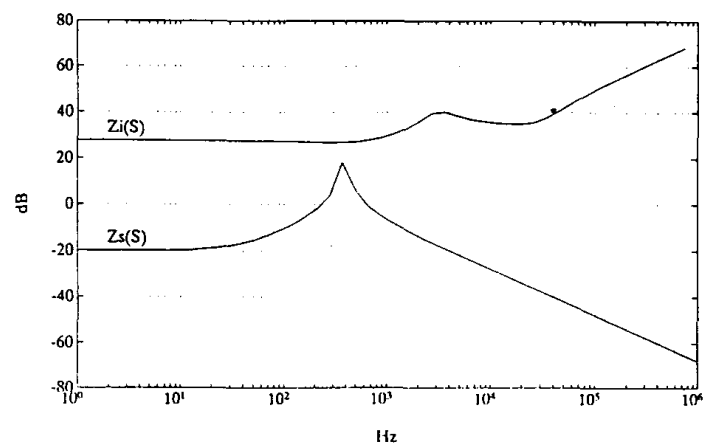

Figure 7 Magnitude of $\mathrm{Zi}$ and $\mathrm{Zs}$

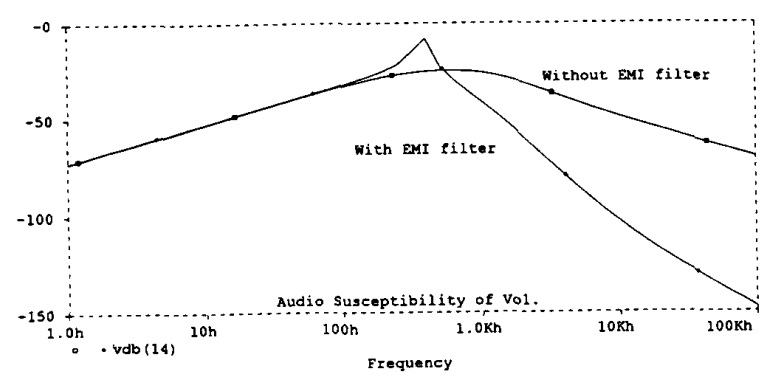

Figure 8 Audiosusceptibility of output \#1

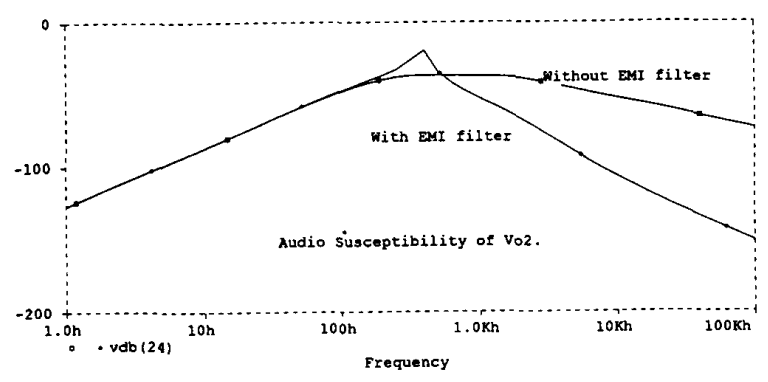

Figure 9 Audiosusceptibility of output \#2

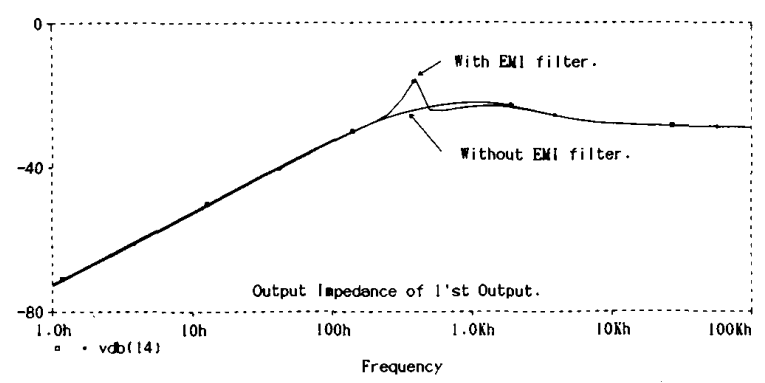

Figure 10 Output impedance of output \#1

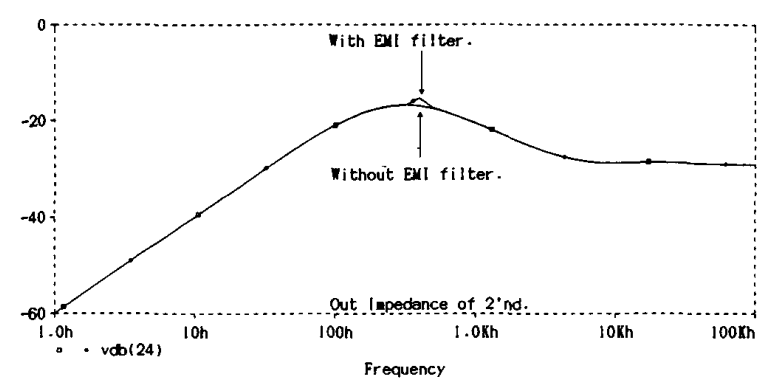

Figure 11 Output impedance of output \#2 


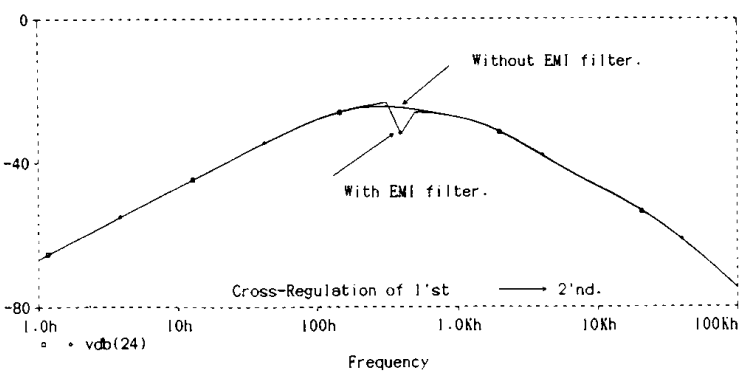

Figure 12 Cross regulation of output \#1 to output \#2

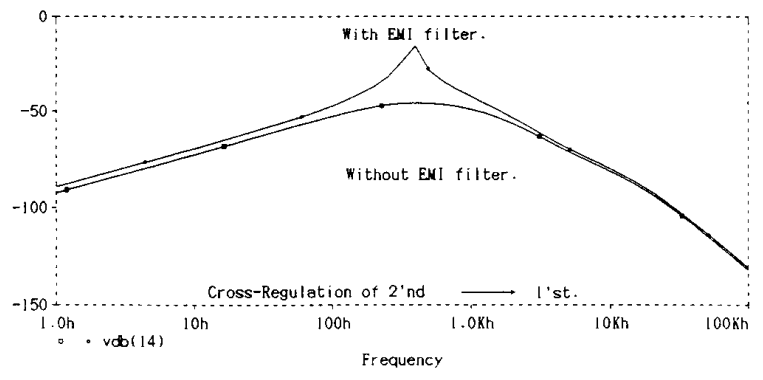

Figure 13 Cross regulation of output \#2 to output \#1

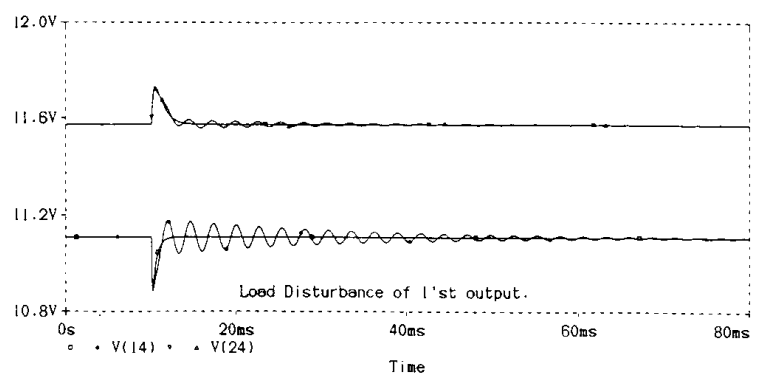

Figure 14 Transient response of output \#1 to the step change of load

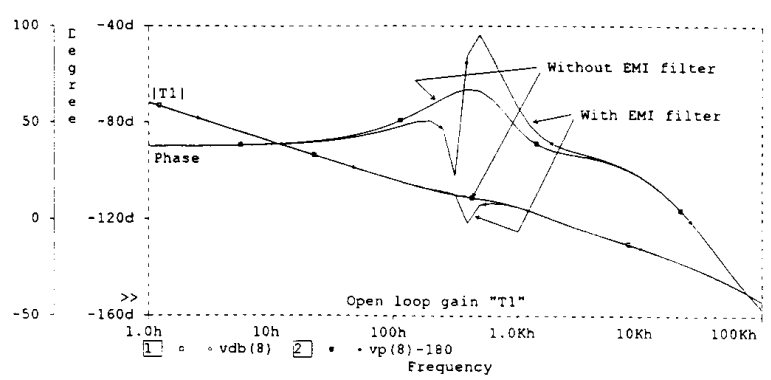

Figure 15 Open loop gain T1

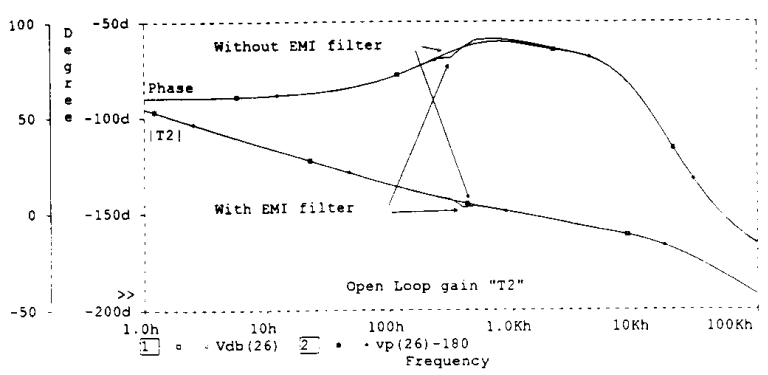

Figure 16 Open loop gain T2

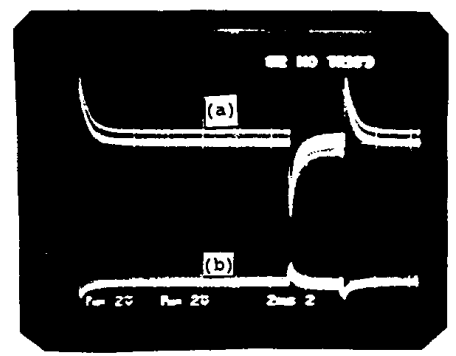

Figure 17 Transient response of the original system to the load variation $(2 \mathrm{~A}-8 \mathrm{~A})$

(a) Output \#1 (b) Output \#2

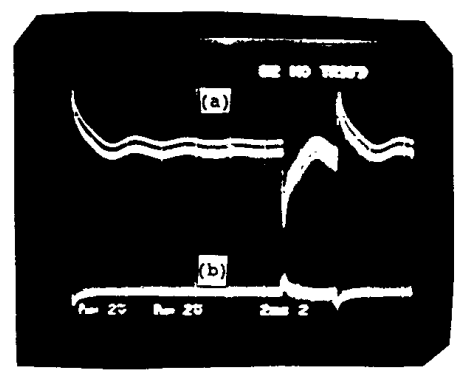

Figure 18 Transient response of the new sytem to the load variation $(2 \mathrm{~A}-8 \mathrm{~A})$ (a) Output \#1 (b) Output \#2 